diesem Element zu übertriebener Wirkung; das Licht des Bewußtseins hiervon hat eine antiseptische Wirkung auf Vorurteile."

Diese Erkenntnisse über die Natur und Herkunft richterlicher Entscheidungen haben eine durchaus praktische Pointe, auf die es hier besonders ankommt: Die Grundrechte lassen sich nicht mit Allgemeinheiten, mit Grundsätzen und mit theoretischer Interpretation verwirklichen, sondern nur durch Entscheidung konkreter Einzelfälle, die ein um sein Recht kämpfender Mensch vor Gericht bringt. Nur dadurch wird das Recht ernst genommen; nur dadurch dringt es auch ins allgemeine Bewußtsein. Als das Bundesverfassungsgericht in den fünfziger Jahren und Anfang der sechziger Jahre seine ersten verdienstlichen Entscheidungen von Einzelfällen, insbesondere den Fall Lüth betreffend Meinungsfreiheit, getroffen hatte, meldete sich als Wortführer der akademischen Verfassungsrechtler Professor Bettermann, Berlin, in der "Juristenzeitung "von 1964 : Das Bundesverfassungsgericht solle nicht Kasuistik und Fallentscheidung an Stelle von Verfassungsinterpretation treiben. Das heißt mit anderen Worten: die Grundrechte sollten im in Deutschland herkömmlichen Sumpf der Abstraktion stecken bleiben. Leider hat sich das Bundesverfassungsgericht in den siebziger Jahren in ein paar Fällen von der eigenen Entscheidung abbringen lassen, ob das Grundrecht wirklich verletzt war oder nicht. Es hat sich damit zufrieden gegeben, zu prüfen, ob das Gericht, dessen Urteil ihm vorlag, "grundsätzlich « die richtige Auffassung vom Grundrecht gehabt habe. Dann komme es nicht mehr darauf an, ob das Grundrecht »möglicherweise « verletzt sei. Das ist unter anderem geschehen in dem von der Wirklichkeit so peinlich überholten Fall des Romans "Mephisto«. Mit dem, was ich aus der amerikanischen Verfassungsjustiz vorgetragen habe, wollte ich davon überzeugen, daß es auf nichts anderes als gerade darauf ankommt. Die Grundrechte werden nur verwirklicht, wenn das Gericht, das zu ihrer Wahrung eingesetzt ist, im Einzelfall darüber entscheidet, ob sie verletzt worden sind.

Richard Schmid

\title{
Kritik von Richtern an Polizeimaßnahmen
}

\section{Eine Dokumentation}

Auch die bundesrepublikanische Richterschaft ist etwa seit Beginn der siebziger Jahre kein monolithischer Block mehr mit einheitlich konservativ gefärbten Ansichten. A uf die Existenz einer zwar minoritären, aber dennoch unübersehbaren Gruppe kritischer Richter und Staatsanwälte haben Böttcher ${ }^{1}$ und Beer/Bäumer ${ }^{2}$ beispielhaft hingewiesen.

Während besonders in Italien und Frankreich bereits seit etwa is Jahren gesellschaftskritische Justizorganisationen bestehen - magistratura democratica und syndicat de la magistrature -, artikuliert sich in der Bundesrepublik erstmals in einer politisch rückschrittlichen Situation eine Stimme in der Justiz, die sich weder durch Korpsgeist noch durch tradierte Funktionsbestimmungen von Kritik an der Justiz

\footnotetext{
I Böttcher, Zum Selbstverständnis gewerkschaftlich organisierter Richter und Staatsanwälte, KJ2/198ז, S. $172 \mathrm{ff}$.

2 Beer/Bäumer, Richterratschlag, $\mathrm{KJ}_{2} / \mathrm{x}_{9} 82$, S. ${ }_{173} \mathrm{ff}$.
} 
oder gesellschaftlichen Mißständen abhalten lassen will. Die Beispiele in Italien und besonders in Frankreich haben allerdings deutlich gemachr, daß Staats- und Justizapparate zunächst auf veröffentlichte justizinterne Kritik auch in reformoffenen Zeiten repressiv reagieren. ${ }^{3}$ Es steht daher zu befürchten, daß auch hierzulande versucht wird, im Zuge restaurativer Tendenzen richterliche Kritik im Keime zu erstikken.

Die Versuche, kritische Richter disziplinarisch zu gängeln, häufen sich auch tatsächlich in den letzten Jahren. Erinnert sei an die Disziplinar- und das Strafverfahren gegen den Richter am Amtsgericht Hildesheim, Ulrich Vultejus, den Richter am Amtsgericht Lahr, Krebs, der erst beim BGH gegen seinen Justizminister Eyrich (Baden-Württemberg) obsiegte, oder an das noch anhängige Vertahren gegen die Richterin am Landsgericht Regensburg, Erika Simm. Richterin Simm hatte einen sehr kritischen öffentlichen Aufruf gegen die Nürnberger Massenverhaftungen mitunterzeichnet. ${ }^{+}$

Eine Gruppe Frankfurter Richter - vorwiegend aus der Arbeitsgerichtsbarkeit hatte sowohl in einem Leserbrief, der von der Frankfurter Rundschau als Artikel mit dem Titel »Richter schelten Innenminister « veröffentlicht wurde, als auch als Mitunterzeichner eines Aufrufs des »Komitees für Grundrechte und Demokratie« kritisch zum Bau der Startbahn West, zum Vorgehen der Polizei bei Einsätzen und zu öffentlichen Erklärungen des hessischen Innenministers Stellung genommen. ${ }^{5}$ Dieses Verhalten führte zu zwei kleinen parlamentarischen Anfragen von CDU- und einem FDP-Abgeordneten, die u. a. das Ziel verfolgten, den zuständigen Sozialminister zu disziplinarischen Maßnahmen gegen die betreffenden Richter zu veranlassen. ${ }^{6}$

Gemeinsam ist allen genannten Verfahren, daß ein Verhalten, das herkömmlich als Sakrileg unter Richtern gilt, nämlich öffentlich geäußerte Kritik, bestraft werden sollte. Den juristischen Aufhänger zur Disziplinierung sah man in $₫ 39 \mathrm{DRiG}$, dem sogenannten Mäßigungsgebot. Diese Vorschrift, die vom Richter im Interesse der Rechtssuchenden auch außerdienstlich ein Verhalten fordert, das deren Vertrauen in die Unabhängigkeit des Richters nicht gefährdet, wird dabei allerdings i. d. R. entsprechend der gängigen Pervertierung von subjektiven Schutzrechten gegenüber dem Staat zu einer Staatsschutznorm uminterpretiert. Nicht mehr der Bürger und potentielle Verfahrensbeteiligte in einem konkreten Verfahren sind Adressaten eines befürchteten Vertrauensverlusts, sondern der Staat selbst, der jede private Kritik durch selbst betroffene Richter - im konkreten Fall als aktive Startbahngegner und als solche vom Innenminister angegriffene Personen - unterbinden möchte.

Die nachstehenden Dokumente machen die genannte Zielsetzung deutlich. Während zunächst in der kleinen Anfrage von drei CDU-Abgeordneten in relativ allgemein gehaltenen Formulierungen nach Disziplinarmaßnahmen gefragt wurde, ließ die Anfrage des Abgeordneten Weghorn aus der FDP deutlich erkennen, wie er sich - der sich selbst übrigens gern mit dem Etikett des Reformjuristen schmückt - die zulässigen Verhaltensweisen eines Richters in der Öffentlichkeit vorstellt.

Die klare und knappe Antwort des hessischen Sozialministers - veröffentlicht einen Tag nach der Wahlaussage der FDP für die CDU - ist inso weit erfreulich, als sie sich zu einer restriktiven Auslegung des $\$ 39$ DRiG bekennt und damit klarstellt, daß auch scharf formulierte öffentliche Kritik durch Richter unter den Schutz von Art 5 GG fällt. Eine Wertung, die übrigens auch durch die Entscheidung des Niedersäch-

\footnotetext{
3 Erinnert sei beispielsweise an die Affüren um den Ermittlungsrichter de Charette in Rouen: s. Bäumer, $\mathrm{KJ} 3 / 1976, \mathrm{~S} .278 \mathrm{ff}$.

4 vgl. Beer/Bäumer, a. a. O.

s FR v. 24. 10. 1981 und v. 17. 12. $198 \mathrm{r}$.

6 Drucksachen des Hess. Landtages, 9. W'ahlperiode, 9/6302: 9/575 1
} 
sischen Dienstgerichts in Sachen Ulrich Vultejus bestätigt wird.' Allerdings läßt die Antwort des hessischen Sozialministers nicht erkennen, welche Bewertungsmaßstäbe er bei der Interpretation des $\$ 39 \mathrm{DRiG}$ zugrundelegen will; immerhin hat er aber der Versuchung widerstanden, die eigene Betroffenheit mit einem von $\$ 39$ DRiG geforderten allgemeinen Vertrauensverlust in die Unabhängigkeit der Richter gleichzusetzen.

Bernd Richter

\section{A. Richter gegen Polizeieinsätze auf der Startbahn West}

Antwort des Sozialministers auf die Kleine Anfrage des Abg. Weghorn (F. D. P.) betreffend Vertrauen auf die unbedingte Verpflichtung von Richtern auf Gesetz und Recht - Drucksache $9 / 6302$

Die Kleine Anfrage beantworte ich im Einvernehmen mit dem Minister der Justiz wie folgt:

r. Sechs Frankfurter Richter haben in einem vom Dezember 1981 datierenden offenen Brief an die Hessische Landesregierung u. a. folgende Vorwürfe bzw. Forderungen erhoben:

a) "Durch harte Polizeieinsätze (wurde) ein Klima geschaffen, das verzweifelte Wutausbrüche bei den Demonstranten provozierte.

b) »Mit ... knüppelharten und chemischen Einsätzen der Polizei wurde staatlicherweise ein Weg der Gewalt beschritten, der wiederum Gewalt erzeugt.،

c) »Demonstranten, die in Auseinandersetzungen geraten sind, werden auch dann, wenn ihnen keine konkrete Gewalttaten nachzuweisen sind, mit strafrechtlichen Verfahren überzogen."

d) Wir fordern ... eine Amnestie für alle, die im Zusammenhang mit den Auseinandersetzungen um die Startbahn 18 West straf- bzw. disziplinarrechtlich belangt werden.* Zu a) und b):

Teilt die Landesregierung meine Auffassung, daß diese Aussagen eine agitatorische Verdrehung von 'Tatsachen beinhalten, und daß dieser Umstand wie auch die Radikalität der Sprache geeignet sind, Zweifel an einem am Gerechtigkeitsgedanken orientierten Urteilsvermögen der betreffenden Richter zu erzeugen, und ferner geeignet sind, dem Ansehen der Rechtspflege in der Öffentlichkeit insgesamt Schaden zuzufügen?

Zu c):

Teilt die Landesregierung meine Auffassung, daß hierin der Vorwurf des Rechtsmißbrauchs von seiten anderer Organe der Rechtspflege enthaiten ist?

$\mathrm{Zu} \mathrm{d):}$

Teilt die Landesregierung meine Auffassung, daß eine Amnestieforderung durch Richter geeignet ist, Zweifel an der Unparteilichkeit von Mitgliedern der Rechtspflege zu erzeugen?

$\mathrm{Zu} \mathrm{I:}$

Mir liegt ein bei dem Ministerpräsidenten im Dezember 1981 eingegangener sog. "Offener Brief " vom 17. Dezember 198I vor, der die genannten Zitate im wesentlichen enthält, jedoch lediglich die Unterzeichnernamen von ca. 40 Personen - darunter zwei Richtern an Arbeitsgerichten - mit dem Zusatz "weitere 380 Unterzeichner « aufführt. Daneben ist mir ein in der Frankfurter Rundschau vom 29. Januar 1982 als Anzeige veröffentlichter „Offener Brief» bekannt, der als Unterzeichner auch die Namen weiterer Richter an Arbeitsgerichten nennt. Dieser veröffentlichte Brief weicht jedoch teilweise von dem erstgenannten Offenen Brief ab. So ist in der Anzeige z. B. hinsichtlich des Zitats zu b) nicht von »knüppelharten und chemischen Einsätzen der Polizei« die Rede, sondern lediglich von »Einsätzen der Polizei«. Das unter c) genannte Zitat ist hierin nicht enthalten. 
Zu a) und b):

Das auch dem Richter zustehende Grundrecht der freien Meinungsäußerung schließt das Recht mit ein, politisch motivierte Äußerungen kundzutun und an staatlichem Verhalten Kritik zu üben. Allerdings findet dieses Grundrecht seine Schranken in den Vorschriften der allgemeinen Gesetze. Hierzu gehört auch $\$ 39$ des Deutschen Richtergesetzes, wonach sich der Richter auch bei politischer Tätigkeit so zu verhalten hat, daß das Vertrauen in seine Unabhängigkeit nicht gefährdet wird. Die Schranken, die $\$ 39$ des Deutschen Richtergesetzes dem Richter bezüglich der freien Meinungsäußerung setzt, halte ich bei Würdigung der Zitate noch nicht für überschritten.

Demgemäß beantworte ich die Frage insoweit mit Nein.

Ungeachtet dessen habe ich im Hinblick darauf, daß in der Öffentlichkeit das Thema Startbahn i 8 West nunmehr seit Monaten teils sehr kontrovers, emotional aufgeladen und unterschiedlich motiviert diskutiert wird, den Präsidenten des Landesarbeitsgerichts gebeten, die Richter der Arbeitsgerichtsbarkeit besonders auf den Grundsatz des $\$ 39$ des Deutschen Richtergesetzes hinzuweisen.

Zu c):

Nein.

Ich unterstelle Richtern die Kenntnis, daß Ermittlungsverfahren nicht erst bei Nachweis konkreter Gewalttaten, sondern nach $\$ 160$ StPO schon bei dem Verdacht einer Straftat eingeleitet werden. Insoweit kann das Zitat, wie auch andere Formulierungen, zu Mißverständnissen führen. Dabei gehe ich davon aus, daß der Offene Brief von Dritten formuliert war.

Zu d):

Die Forderung nach Amnestie ist eine politische Forderung. Der Richter hat sich auch bei politischer Betätigung so zu verhalten, daß das Vertrauen in seine Unabhängigkeit nicht gefährdet wird. Das Zitat enthält eine sehr allgemein gehaltene Amnestieforderung, die zudem erst einmal die rechtskräftige Verhängung von Straftaten voraussetzt. Unter diesen Geischtspunkten halte ich die dem Richter im Interesse der Wahrung seiner Unabhängigkeit gezogenen Verhaltensgrenzen noch nicht für überschritten.

2. In einem Brief an die Frankfurter Rundschau (veröffentlicht am 24. Oktober I98 r, Seite 14) haben zwölf hessische Arbeitsrichter unter anderem ausgeführt: (Der Innenminister wird aufgefordert,) "Zur Kenntnis zu nehmen, daß zu den zeitweilig im Hüttendorf Anwesenden oder Unterstützern der Bewohner neben den "Vorzeigebürgern« aus Walldorf auch etliche Richter des Landes, wie zum Beispiel die Unterzeichner, gehören.»

Wie bewertet die Landesregierung die Tatsache, daß sich Richter nicht nur an rechtswidrigen Handlungen beteiligen, sondern sich auch noch offentlich und unter Verweis auf ihr Richteramt dazu bekennen?

3. Welche Schritte hat die Landesregierung in dieser Angelegenheit unternommen?

Hierzu verweise ich auf meinen Schriftlichen Bericht zu dem Berichtsantrag der Abg. Stanitzek, Lenz (Frankfurt), Koch, Nassauer (CDU) und Fraktion betreffend Beteiligung hessischer Richter am ehemaligen Hüttendorf auf dem geplanten Gelände der Startbahn 18 West in Frankfurt am Main - Drucks. 9/575 I -, den ich als Anlage beifüge.

Ergänzende Informationen liegen mir hierzu nicht vor.

Wiesbaden, den 28. Mai 1982

Clauss

[Hess. Landtag, 9. Wahlperiode, Drucksache 9/660I] 


\section{B. Richter im Hüttendorf}

Schriftlicher Bericht des Hessischen Sozialministers im Einvernehmen mit dem Minister des Innern und dem Minister der Justiz zum Berichtsantrag der Abg. Stanitzek, Lenz (Frankfurt), Koch, Nassauer (CDU) und Fraktion betreffend Beteiligung hessischer Richter am ehemaligen Hüttendorf auf dem geplanten Gelände der Startbahn 18 West in Frankfurt am Main - Drucksache 9/575 I

I. Treffen Pressemeldungen (vgl. Frankfurter Rundschau vom 24. Oktober 198r) zu, wonach zu den zeitweilig im Hüttendorf Anwesenden oder Unterstützern der Bewohner auch etliche Richter des Landes Hessen gehörten?

Mir liegt eine Pressemeldung der Frankfurter Rundschau vor, aus der hervorgeht, daß jedenfalls auch $\mathbf{2}$ hessische Richter an Arbeitsgerichten zu den im Hüttendorf zeitweilig Anwesenden gehörten. Die Frankfurter Rundschau zitiert in dem genannten Artikel Auszüge aus einem an sie gerichteten Brief dieser Richter, in dem diese sich kritisch mit Äußerungen von Staatsminister Gries auseinandersetzen. Der genaue Wortlaut des Briefes ist mir nicht bekannt.

2. Wenn ja, sind der Landesregierung die Namen der betreffenden Richter bekannt?

Vier Richter an Arbeitsgerichten haben dem Präsidenten des Landesarbeitsgerichts gegenüber gesprächsweise erwähnt, daß sie zu den Unterzeichnern des genannten Briefes gehören. Einer davon hat ihm mitgeteilt, daß er den Hüttendorfbewohnern finanzielle Zuwendungen gemacht habe.

3. In welcher Art und Weise haben sich die betreffenden Richter an Aktionen der Startbahngegner beteiligt?

Über den genannten Artikel und über die Angaben zu Frage 2 hinaus liegen mir in dieser Angelegenheit keine Informationen vor. Es ist mir daher - von der erwähnten finanziellen Zuwendung abgesehen - auch nicht bekannt, ob und in welcher Weise sich die betreffenden Richter an Aktionen der Startbahngegner beteiligt haben.

4. Hat die Landesregierung auf Grund der Aktivitäten der betreffenden Richter Disziplinarmaßnahmen gegen diese eingeleitet, und wenn ja, welche?

Nein

5. Wenn nein, warum nicht?

Der mir bekannte Sachverhalt vermag die Einleitung von disziplinarischen Maßnahmen nicht zu rechtfertigen.

Clauss

[Hess. Landtag, 9. Wahlperiode, Drucksache 9/5751] 